\title{
Ondeletas en ingeniería. Principios y aplicaciones
}

\author{
V. Saavedra-Gastélum ${ }^{\boxplus 2)}$, T. Fernández-Harmony ${ }^{3)}$, T. Harmony-Baillet ${ }^{4)}$ y V.M. Castaño-Meneses ${ }^{5)}$ \\ Universidad Autónoma de Q uerétaro, División de estudios de Posgrado y Centro de Física A plicada y Tecnología \\ A vanzada, UN A M , Campus Juriquilla, Q uerétaro ${ }^{122}$, Instituto de N eurobiología, U N A M , Campus Juriquilla, \\ Q uerétaro ${ }^{3 / 4}{ }^{4}$, Centro de Física A plicada y Tecnología A vanzada, UN A M , Campus Juriquilla, Q uerétaro ${ }^{5)}$ \\ E-mails: verosaavedra@ fata.unam.mx, thalia@servidor.unam.mx, castano@ fata.unam.mx
}

(Recibido: abril de 2005; aceptado: diciembre de 2005)

\begin{abstract}
Resumen
La transformada de ondeleta es una función matemática que permite seccionar los datos en pequeños componentes en escala frecuencia-tiempo y analizarlos cada uno por separado. Su mayor ventaja sobre la transformada de Fourier es que las ondeletas permiten trabajar con datos que presentan discontinuidades o picos. El concepto de ondeleta surgió de manera independiente en diversas áreas como la física cuántica, matemáticas, ingeniería eléctrica, etc. Este artículo pretende dar a conocer al lector el concepto básico de transformada de ondeleta, sus fundamentos, así como algunas de sus aplicaciones.
\end{abstract}

Descriptores: Ondeletas, frecuencia-tiempo, FFT, espacio fase, no-estacionaria, ondeleta madre

\begin{abstract}
Wavelets are math e mat i cal functions that cut up data into differ ent frequency com po nents, and then study each com ponent with a res olu tion matched to its scale. They have ad van tages over tra di tional F ou rier meth ods in an a lyzing phys i cal sit $u$ a tions wherethesig nal con tains dis con ti nu ities and sharp spikes. Wavelets were devel oped in de pend ently in thefields of mathematics, quan tum physics, elec tri cal en gi neer ing, etc. This ar ti clepretends to givethe researcher an over view about ba sic wavelets con cepts, as well as, thefun da men tal theory and application of wavelets
\end{abstract}

Keywords: Wave lets, frequency-time, FFT, phase space, nonstationary, main wavelets.

\section{Introducción}

A través de los tiempos, se ha buscado una manera eficaz de representar distinto tipo de señales. Hasta hace poco, para analizar señales complejas se utilizaban principalmente métodos de series de tiempo, en particular, análisis espectrales de las potencias, como es el caso del conocido análisis de Fourier. En general, todos los análisis espectrales descomponen una forma de onda compleja en una suma lineal de componentes de onda más elementales; en el caso particular del análisis de Fourier, las ondas ele- mentales son seno y coseno. Al aplicar la transformación lineal, la señal que está en el dominio del tiempo cambia a otra función en el dominio de las frecuencias. Sin embargo, estos análisis se basan en supuestos que muchas veces las señales no satisfacen. La Transformada de Fourier presupone que la señal tenga ciertas características, entre ellas, la estacionalidad. Habitualmente, este supuesto es violado, es decir, se aplica la transformación de Fourier a señales que no son estacionarias, de modo que el resultado pudiera no representar la realidad. Problemas de este tipo son los que han motivado la búsqueda de otras formas 
Ondeletas en ingeniería. Principios y aplicaciones

de análisis de señales, como las llamadas ondeletas.

Las ondeletas son un descubrimiento relativamente nuevo en las matemáticas aplicadas. Deben su nombre a los trabajos de Morlet, Arens, Fourgeau y Giard (1982), Morlet (1983), Grossman y Morlet (1984), (Daubechies, 2004). El interés por las ondeletas ha crecido en las últimas dos décadas por diferentes razones. Por un lado, el concepto de ondeletas puede verse como una síntesis de ideas que se originaron en los últimos treinta años en el área de la ingeniería, de la física y de las matemáticas puras, por otro lado, las ondeletas proveen una herramienta matemática muy sencilla con una gran variedad de aplicaciones. Por ejemplo, en el análisis de señales de sonido e imagen se pueden encontrar en (Daubechies, 2004) a Kronland-Martinet, Morlet y Grossmann (1987), Mallat (1989); y en análisis numérico a Beylkin, Coifman y Rokhlin (1991). La transformada de ondeleta aparece, entonces, como una necesidad de analizar funciones no estacionarias en su escala de frecuencia-tiempo.

Las ondeletas se utilizan fundamentalmente para analizar funciones, de acuerdo al método de escalamiento. La transformada de ondeleta es una función que satisface ciertos requerimientos matemáticos que se utilizan para representar datos 0 funciones.

En la transformada de ondeleta, la escala juega un papel muy importante, dicho proceso analiza los datos en diferentes escalas o resoluciones. Es decir, si se toma una "ventana" de los datos, notaríamos el mismo comportamiento que si se toma una ventana más pequeña, y así sucesivamente.

La transformada de ondeleta provee una representación en frecuencia-tiempo simultáneamente, corta la señal de interés en varias partes y analiza cada una de ellas por separado. Las frecuencias altas o contraídas pueden ser mejor localizadas en el tiempo y las frecuencias bajas 0 dilatadas en la frecuencia.

La transformada de ondeleta, al igual que la transformada de Fourier, es una transformación reversible, que permite ir de la señal original a la transformada y viceversa, cuando se requiera.

La transformada de ondeleta trabaja en el espacio fase, lo que significa que se usa un espacio conformado simultáneamente por coordenadas de tiempo y frecuencia. Esto nos permite hacer operaciones mediante desplazamientos en coordenadas, en vez de trabajar con ecuaciones diferenciales o ecuaciones algebraicas.

\section{Antecedentes históricos}

El concepto de ondeletas es relativamente nuevo, de hace no más de 20 años; sin embargo, los esfuerzos por analizar señales se remontan al año de 1807, con los trabajos de Joseph Fourier acerca del análisis de frecuencias, lo que dio origen a las Series de Fourier. En 1930, diversos investigadores enfocaron su esfuerzo a la representación de funciones utilizando funciones básicas de escala variable llamadas Funciones básicas de Haar.

La transformada de Fourier hizo posible la descomposición de cualquier función periódica en un conjunto de funciones bases ortonormales de senos y cosenos, lo cual permite analizar la señal en términos de su frecuencia.

En 1909, Alfred Haar descubrió otro sistema ortonormal de funciones. La investigación de Haar proporcionó la forma más simple de ondeletas ortonormales que consistía en un conjunto de funciones base rectangulares. El uso de las funciones básicas de Haar quedaba limitado, debido a su discontinuidad.

La teoría expuesta por Haar, fue enriquecida en la década de los treinta por Levey quien utilizó las bases de Schauder para examinar propiedades de regularidad locales, las cuales eran imposibles de estudiar a través de la transformada de Fourier. En 1946, Dennis Gabor intro dujo las ondeletas Gabor. Su idea principal era cortar una onda en segmentos de onda (ondeletas) donde cada segmento tuviera bien definida su banda de frecuencias y su posición en el tiempo. La ondeleta de Gabor presentaba un gran avance para descomponer una señal continua; sin embargo, presentaba problemas en la descomposición de señales discretas. 
Entre 1960 y 1980, los matemáticos Weiss Guido y Coifman Ronald, estudiaron los elementos más simples del espacio de función llamados átomos y encontraron las reglas de ensamble, las cuales permiten la reconstrucción de to dos los elementos de un espacio de función a partir de esos átomos. Entre 1980 y 1990, Grossman y Morlet utilizaron las ondeletas para analizar temblores y modelar el proceso de las ondas del sonido viajando a través de la corteza terrestre. Aplicaron por primera vez las ondeletas para modelar funciones no estacionarias.

En 1985, Mallat Stephane impulsó el desarrollo de las ondeletas a partir de su trabajo en el procesamiento de señales digitales, ya que descubrió relaciones entre los filtros de la cuadratura del espejo, algoritmos piramidales y bases de ondeletas ortonormales. Inspirándose en parte de estos resultados, Yves Meyer construyó las primeras ondeletas no triviales, las cuales son diferenciables; sin embargo, no decaen rápidamente a cero, lo que es necesario para la convergencia de las series que se forman a partir de ellas. Años más tarde, Daubechies Ingrid (2004), utilizó el trabajo de Mallat y construyó un conjunto de funciones base de ondeletas ortonormales que se convirtieron en la piedra angular de la aplicación de ondeletas en nuestros días.

\section{Elementos básicos de transformadas de ondeletas}

La transformada de ondeleta, vista como un proceso matemático, es una operación lineal que descompone una señal compleja en bloques elementales que aparecen en diferentes escalas 0 resoluciones. Dichos bloques pueden ser generados de una forma muy sencilla, lo cual simplifica su análisis, permitiendo a su vez, limpiar la señal de interferencia externa.

La selección de la mejor ondeleta depende de las características propias de la señal de estudio; entonces, el punto clave es encontrar la mejor función base. A esta función se le conoce como ondeleta madre.

La función base seleccionada contiene información sustancial acerca de la señal. Si esta función base describe bien a la señal en términos de que está dada la eficiencia, entonces sólo serán necesarios algunos términos en la expansión para representar la señal completa. Es importante mencionar que sólo aquellas funciones que satisfagan las dos condiciones siguientes pueden ser consideradas como ondeleta madre:

\section{Ser oscilatorio (área total igual a cero)}

\section{Decaer a cero rápidamente}

La transformada de ondeleta de una señal continua está definida por:

$$
C W T_{\mathrm{y}} x(a, b)=W_{x}(a, b)=\bigoplus_{-:}^{¥} x(t) \mathrm{y}_{\mathrm{a}, \mathrm{b}}^{*}(t) d t
$$

y

$$
\mathrm{y}_{a, b}(t)=|a|^{-1 / 2} \underset{\mathrm{y}}{\hat{\mathrm{e}} t} \frac{\hat{\mathrm{C}}-b}{a} \emptyset
$$

donde la señal $x(t)$ es transformada por la ondeleta madre. La función ? $(t)$ se dice que es una ondeleta si y sólo si su transformada de Fourier $\hat{\mathbf{y}}(w)$ satisface:

$$
\underset{¥}{¥} \frac{\mid \hat{y}(w)^{2}}{|w|} c_{y}<¥
$$

La condición de admisibilidad implica que

$$
\stackrel{¥}{¥} \mathrm{y}(\mathrm{t}) \mathrm{dt}=0
$$

lo que significa que ?(t) es oscilatoria y su área efectiva es cero. El factor:

$$
a^{-172}
$$

es utilizado para normalizar la energía. El parámetro a controla la dilatación o la contracción de la función en la escala del tiempo y amplitud, y el parámetro b controla la traslación de la función en el tiempo.

El procedimiento consiste en adoptar la función prototipo de ondeleta y por dilatación descomponer la señal en escalas diferentes con distintos niveles de resolución. Como la transformación es alcanzada a partir de dilatar y 
Ondeletas en ingeniería. Principios y aplicaciones

trasladar la ondeleta madre continuamente sobre los números reales, se genera información redundante. Por lo tanto, para quitar dicha información, la ondeleta madre puede ser dilatada y trasladada de manera discreta haciendo $a=a_{0}^{m}$ y $b=n b_{0} a_{0}^{m}$, donde $a_{0}$ y $b_{0}$ son constantes fijas con $a_{0}>1$, $b_{0}>0, m, n \in \mathbf{Z}$ ( $\mathbf{Z}$ en el conjunto de enteros positivos). Entonces, la ondeleta madre discreta y su correspondiente transformada de ondeleta se convierten en:

$$
D W T_{\mathrm{y}} x(m, n)=\bigoplus_{-¥}^{¥} x(t) \mathrm{y}_{m, n}^{*}(t) d t
$$

y

$$
y_{m, n}(t)=\left|a_{0}\right|^{-m / 2 / 2} \frac{\hat{E} t-n b_{0} a_{0}^{m} \ddot{o}}{a_{0}^{m}} \varnothing
$$

Sea $x(t)$ una secuencia discreta, entonces la ecuación (3) se convierte en

$$
D W T[m, n]=\frac{1}{\sqrt{a_{0}^{m}}} \hat{A}_{k} x[k] y\left[a_{0}^{-m} n-k\right]
$$

\section{Aplicaciones: algunos ejemplos}

\section{Huellas dactilares en el FBI}

Desde 1924 hasta nuestros días, el FBI ha recolectado alrededor de 30 millones de huellas dactilares. El archivo consiste principalmente en impresiones de tinta sobre papel. Dichas impresiones fueron distribuidas a través de faxes a diversas agencias de la fuerza pública, pero la calidad de la digitalización es muy baja. Algunas jurisdicciones están experimentando tener un almacenamiento digital de las huellas dactilares, lo cual ha generado un problema de incompatibilidad de formato en los datos. Debido a este problema, la comunidad de justicia criminal está interesada en estandarizar la digitalización y la compresión de los datos.

En 1993, el FBI creó los procedimientos para estandarizar la digitalización y compresión de huellas dactilares en colaboración con el Instituto Nacional de Tecnología y Estándares, el Labora- to rio Nacional de los Álamos, comerciantes y la comunidad de justicia criminal.

El problema en perspectiva es el siguiente: las imágenes de las huellas dactilares son digitalizadas a una resolución de 500 píxeles por pulgada con 256 niveles de gris por píxel. Una sola huella dactilar contiene 700,000 píxeles y necesita $0.6 \mathrm{MB}$ de espacio en disco duro. Un par de manos para cada persona requiere $6 \mathrm{MB}$ de espacio. Por lo tanto, digitalizar el actual archivo del FBI necesitaría 200 tetrabytes de espacio, lo cual implicaría un costo de 200 millones de dólares. Obviamente, el uso de la transformada de ondeletas para la compresión de datos es necesario en este caso.

\section{Tonos M usicales}

Wickerhauser Victor, sugiere que las ondeletas pueden ser útiles para la síntesis del sonido. Su idea es que un generador de paquetes de onda sencillo pueda reemplazar a un largo número de oscilaciones. A través de la experimentación, un músico puede determinar combinaciones de paquetes de onda y producir sonidos interesantes.

Wickerhauser siente que la síntesis de sonidos es una aplicación natural de las ondeletas. Digamos que se desea simular los sonidos de los instrumentos musicales, entonces una muestra de notas producidas por un instrumento puede ser descompuesta en su paquete de coeficientes de ondeleta, de modo que, para simular una nota, se requerirá recargar los coeficientes dentro del paquete generador de ondeletas y reproducir el resultado.

Algunas características de la música como las variaciones en intensidad, de cómo empieza y cómo termina un sonido, pueden ser controladas de manera independiente. Se pueden utilizar paquetes de onda larga y codificar estas propiedades incluso para cada una de las notas. Cualquiera de estos procesos puede ser controlado en tiempo real utilizando, por ejemplo, un teclado. Nótese que el instrumento musical puede ser incluso la voz y las notas, palabras o fenómenos. 
V. Saavedra-Gastélum, T. Fernández-Harmony, T. Harmony-Baillet y V.M. Castaño-Meneses

Un sintetizador de música con un generador de paquetes de ondeleta puede almacenar muchos sonidos complejos eficientemente, debido a que:

- Los paquetes de coeficientes de ondeleta, como los coeficientes de ondeleta, son en su mayoría muy pequeños para muestras digitales de señales suaves.

- Los coeficientes de desecho que salen por debajo de una banda predeterminada con anterioridad, introducen sólo pequeños errores cuando se comprimen los datos de señales suaves.

- Similarmente, un sintetizador de paquete de onda puede utilizarse para reconstruir señales muy comprimidas.

A pesar de que las descargas parciales sólo envuelven pequeños montos de energía, pueden llevar al deterioro progresivo de las propiedades dieléctricas de materiales de aislamiento en transformadores, generadores y motores. La medición en el equipo actual es complicada, debido a la disminución, resonancia y el fenómeno de onda.

Las descargas parciales ocurren en forma de pulsaciones eléctricas individuales, las cuales pueden ser detectadas como señales eléctricas en un circuito externo conectado al objeto 0 aparato probado.

Las descargas eléctricas pueden ocurrir en cavidades 0 impurezas en materiales sólidos de aislamiento, en burbujas de gas, en líquido de aislamiento 0 entre caspas de aislamiento con diferentes características dieléctricas.

Los propósitos de medir las señales de descargas parciales son los siguientes:

1. Para verificar que el equipo probado no presente descargas parciales mayores a una magnitud especificada arbitrariamente dado un cierto voltaje.

2. Para determinar la amplitud del voltaje, en donde las descargas parciales de una magnitud específica baja comienza al incrementarse el voltaje y cesa cuando el voltaje disminuye.

3. Para determinar la magnitud de la cantidad específica de descargas parciales en un cierto voltaje específico.

La magnitud y el tiempo de ocurrencia de las descargas parciales son almacenados en una computadora por un determinado período de tiempo, por lo tanto, se puede obtener la magnitud vs el tiempo de ocurrencia y la fase vs la distribución de ocurrencia. Sin embargo, to das las metodologías actuales tienen que lidiar con el problema del ruido.

En 1997, Ramírez-Niño, Rivera-Castañeda, García-Colón y Castaño (1998), utilizaron el análisis de transformada de ondeletas para reducir el ruido y detectar el tiempo relativo en que ocurrían las descargas parciales en señales eléctricas y acústicas en materiales de aislamiento. Encontraron que la aproximación mediante ondeletas resulta ser poderosa en la reducción de ruido para este tipo de señales.

\section{Conclusiones y perspectivas}

Como ya se ha visto, la transformada de ondeleta resulta ser una herramienta matemática muy poderosa para la resolución de problemas relacionados con señales de todo tipo, desde mecánicas hasta biológicas. Nos permite a su vez, analizar fenómenos complicados "dividiéndolos" en pequeños componentes que se pueden analizar por partes, haciendo el trabajo mucho más sencillo, además de dar una visión global de cómo las interacciones de sus componentes conforman a un sistema.

Existen diversas áreas de la ingeniería, no exploradas aún, donde la transformada de ondeletas puede aplicarse, tales como el análisis de ondas cerebrales, las turbulencias, la predicción de terremotos, etc. En pocas palabras, las ondeletas seguirán "haciendo olas". 


\section{Referencias}

Daubechies I. (2004). Ten Lectures on Wavelets. $8 a$ ed. Philadelphia: SIAM, CBMS-NSF Regio- nal Conference Series in Applied Mathematics 61.

Ramírez-Niño J., Rivera-Castañeda S., García-Colon V.R., Castaño V.M. (1998). Analysis of Partial Electrical Discharges in Insulating Materials Through the Wavelet Transform. Computational Materials Science, No.9, pp. 379-388.

Weiss $\mathrm{G}$ y Coifman R., Mallat S., Wickerhauser V. (1960-1980), http://www.amara.com/ IEEEwave/IEEEwavelet.html

Haar A. (1909), http://www.wavelet.org/tuto rial/ whistory.htm

\section{Bibliografía sugerida}

Graps A. (1995). An Intro duc tions to Wave lets. IEEE Computational Science and Engineering, Vol. 2, No. 2, Los Alamitos, CA.

Kaiser G., Friendly A. (1994). Guide to Wavelets. Birkhauser, Boston, pp. 44-45.

http://perso.wanado o.fr/polyvalens/clemens/ wavelets/wavelets.html

http://sdcd.gsfc.nasa.gov/ESS/annual.reports /ess97/app/tarek.html

Http://users.rowan.edu/ polikar/WAVELETS/ WTpart1.html

\section{Semblanza de los autores}

Verónica Saavedra-G astélum. Obtuvo su licenciatura en actuaría por el ITAM, México en 1999. Posteriormente, su Master of Sciences in Statistics por la Univer sity of Warwick, Inglaterra en 2001. Le fue otorgado el Diploma “Asesoría y Orientación Educativa" 2002, por el ITESM. Es profesora de cátedra en el ITESM, Campus Querétaro, en la UNAM desde el año 2005, en la UAQ (2006) y en el ITESM, Campus Monterrey (2001-2003). Es asesora independiente en estadística médica desde 2001. Actualmente cursa el segundo semestre del doctorado en ingeniería en la Universidad Autónoma de Querétaro y su línea de investigación es EEG, ondeletas y Kernel.

Thalía Fernández-H armony. Realizó sus estudios de Matemáticas en la Normal Supe rior (Instituto Pedagógico de La Habana, Cuba). Obtuvo la licenciatura en matemáticas, la maestría en neurociencias y el doctorado en Ciencias Fisiológicas, por la Universidad Nacional Autónoma de México. Asimismo, logró un posdoctorado en la Universidad de Cali fornia de San Diego. Ha publicado 55 artículos en revistas de difusión internacional y 10 de difusión nacional, participó en 8 capítulos de libros (4 nacionales y 4 internacionales). Ha sido tutora de 18 tesis, de las cuales 9 están en proceso. Ha recibido las distinciones de SNI (nivel 2), PRIDE (catego ría "D"), y la Medalla Alfonso Caso. Hasta hace 6 años trabajó en la caracterización electroencefalográfica y conductual del electroencefalograma (EEG). Actualmente realiza investigación en Neurorretroalimentación.

Thalía H armony-Baillet. Realizó su carrera como médico cirujano en la Facultad de Medicina de la UNAM, cuando todavía no existían los posgrados. Durante su carrera, trabajó en el entonces Instituto de Estudios Médicos y Biológicos, ahora Instituto de Ciencias Biomédicas. Realizó su doctorado en el Centro de la Investigación Científica de La Habana, Cuba. A su regreso a México, fue profesor titular C en la entonces ENEP Iztacala, ahora FES Iztacala. Desde 1995, se trasladó al Instituto de Neurobiología de la UNAM en Juriquilla, en donde ha realizado varias investigaciones en psicofisiología, y recientemente en el estudio de los factores de riesgo de daño cere bral pre y perinatales.

Víctor $M$ anuel $C$ astaño-M eneses. Obtuvo la licenciatura en ingeniería física en la Universidad Iberoamericana y realizó sus estudios de posgrado en la Facultad de Ciencias de la UNAM, lo cual le concedió el doctorado en ciencias (Física) en abril de 1985. Después de una estancia posdoctoral de dos años en el IBM Thomas J. Watson Research Center en Nueva York, se reincorporó al Instituto de Física de la UNAM, donde alcanzó el nombramiento de investigador titular $C$ en enero de 1991, adscrito al Departamento de Materia Condensada, en donde permaneció hasta abril de 2002, cuando se crea el Centro de Física Aplicada y Tecnología Avanzada, dependencia a la que pertenece en la actualidad. Ha publicado 421 artículos en revistas listadas en índices internacionales, 176 memorias de congresos nacionales e internacionales, 10 capítulos en libros y un libro de divulgación científica, además de ser co-editor de 4 libros. De acuerdo con un estudio de la Office for Naval Research del gobierno norteamericano, Víctor Castaño es uno de los cinco científicos latinoamericanos más productivos y citados en el campo de la ciencia de los materiales. Sus trabajos tecnológicos y de investigación aplicada han sido reseñados en prácticamente todos los periódicos y canales de TV nacionales y en varias instancias internacionales. Entre los reconocimientos nacionales e internacionales que ha recibido, se cuentan el Premio de la Academia de la Investigación Científica (hoy Academia Mexicana de Ciencias), el Premio de la Organización de Estados Americanos, la Distinción Universidad Nacional para Jóvenes Académicos, etc. 